\title{
Magnetic Field Influence on the Thermal Conductivity of $\mathrm{PrB}_{6}$
}

\author{
P. Priputen ${ }^{a}$, J. ŠeBeK $^{b}$, M. Reiffers ${ }^{a}$, E. ŠAntavá $^{b}$, \\ S. Gabáni ${ }^{a}$, P.A. Alekseev ${ }^{c}$, E.V. Nefeodova ${ }^{c}$, \\ I.P. SADIKOV ${ }^{c}$, N. Shitsevalova ${ }^{d}$ AND K. FlachBART ${ }^{a}$ \\ ${ }^{a}$ Centre of Low Temperature Physics, Institute of Experimental Physics \\ Slovak Academy of Sciences \\ Watsonova 47, 04001 Košice, Slovakia \\ ${ }^{b}$ Institute of Physics, V Holešovičkách 2, 18000 Praha, Czech Republic \\ ${ }^{c}$ Russian Research Centre, Kurchatov Institute \\ 123182, Moscow, Russia \\ ${ }^{d}$ Institute for Problems of Material Science \\ Academy of Sciences of Ukraine, $252680 \mathrm{Kiev}$, Ukraine
}

Results of thermal conductivity measurements on single crystalline $\operatorname{PrB}_{6}$ sample in the temperature range of $2-30 \mathrm{~K}$ and in magnetic field up to $14 \mathrm{~T}$ are presented. The obtained results are discussed in order to estimate the electron, phonon, and magnetic contributions to thermal conductivity. Taking into account the results of electrical resistivity of this compound the temperature dependence of the reduced Lorentz function is determined.

PACS numbers: 05.60.--k, 72.15.Eb

\section{Introduction}

Rare earth hexaborides crystallize in the CsCl-based bcc structure with rare earth ion at each cube corner and boron octahedron at the body center $[1,2]$. $\mathrm{PrB}_{6}$ is a metal, which orders antiferromagnetically at low temperatures. The temperature dependence of heat capacity of $\mathrm{PrB}_{6}$ in zero magnetic field $[3,4]$ shows an extremely large peak at $6.9 \mathrm{~K}$ and a smaller peak at $4.2 \mathrm{~K}$, which reveals two phase transitions in this compound. Neutron scattering data $[3,5]$ indicate a spontaneous incommensurate magnetic ordering below $6.9 \mathrm{~K}$ and below $4.2 \mathrm{~K}$ a coexistence of commensurate and incommensurate phases. At $1.74 \mathrm{~K}$, only the commensurate phase is observed. Electrical resistivity and thermal conductivity 
measurements $[3,4,6]$ in zero magnetic field point to interesting transport properties at low temperatures in this compound. This was our motivation for the investigation of thermal conductivity and electrical resistivity of $\mathrm{PrB}_{6}$ in various magnetic fields.

\section{Experimental details}

$\operatorname{PrB}_{6}$ single crystal was prepared by the floating zone method and cut in $\langle 110\rangle$ direction as parallel-piped. We have measured the thermal conductivity $\kappa(T)$ of this sample in the temperature range of $2-30 \mathrm{~K}$ along the $\langle 110\rangle$ direction and in magnetic fields up to $14 \mathrm{~T}$. The magnetic field was oriented parallel to the direction of the sample. Measurements have been performed using the Thermal Transport Option of the PPMS 14, Quantum Design, USA, in which the thermometers and heater setup was modified. The modified experimental procedure was checked by SRM 8420 etalon, NIST.

To estimate the electron contribution of $\kappa(T)$ and the temperature dependences of reduced Lorentz number, electrical resistivity measurements $\rho(T)$ up to $5 \mathrm{~T}$ were made under the same conditions as mentioned above. They were realized by the standard four-probe method in a ${ }^{4}$ He flow cryostat.

\section{Results and discussion}

Temperature dependences of thermal conductivity $\kappa(T)$ of $\operatorname{PrB}_{6}$ in magnetic fields up to $14 \mathrm{~T}$ are shown in Fig. 1a. The values of $\kappa(T)$ in zero magnetic field are similar to those of Ref. [6]. However, as can be seen from Fig. 1a, a large peak of $\kappa(T)$ at $T_{\mathrm{N} 1}$ occurs, while in [6] only a small change of $\kappa(T)$ was observed. With decreasing temperature there is a plateau of $\kappa(T)$ between $T_{\mathrm{N} 1}$ and $T_{\mathrm{N} 2}$. A sharp increase at $T_{\mathrm{N} 2}$ corresponds well with data published in [6]. Then, a gradual increase in $\kappa(T)$ continues down to the lowest experimental temperature in contrary to [6], where a maximum at about $3 \mathrm{~K}$ occurs. However, we expect that it appears at lower temperatures.

The electronic contributions $\kappa_{\mathrm{e}}$ to $\kappa(T)$, being determined from the electrical resistivity at $B=0 \mathrm{~T}$ and $B=5 \mathrm{~T}$ by means of the Wiedemann-Franz law $\left(L_{0}\right)$, are displayed as full lines on the left side of Fig. 1a. According to them we suppose that the main contribution to thermal conductivity of $\mathrm{PrB}_{6}$ comes from the electronic contribution $\kappa_{\mathrm{e}}$. Above $T_{\mathrm{N}}$ the electrons are strongly scattered by phonons and by spin disorder, below $T_{\mathrm{N}}$ by magnons. Therefore, the experimentally measured values of the thermal conductivity $\kappa$ are much smaller than those determined by the Wiedemann-Franz law, $\kappa_{\mathrm{e}}$. At lowest temperatures, when scattering of electrons by phonons and magnons is gradually diminished and the electrical resistivity reaches the residual resistivity value $\rho_{0}$, the thermal conductivity starts to increase and reaches the value $\kappa \doteq \kappa_{\mathrm{e}}$. Therefore, we suppose the appearance of a maximum of $\kappa(T)$ and a decreasing dependence towards zero, below this temperature. A similar behaviour of $\kappa(T)$ is observed at magnetic field 


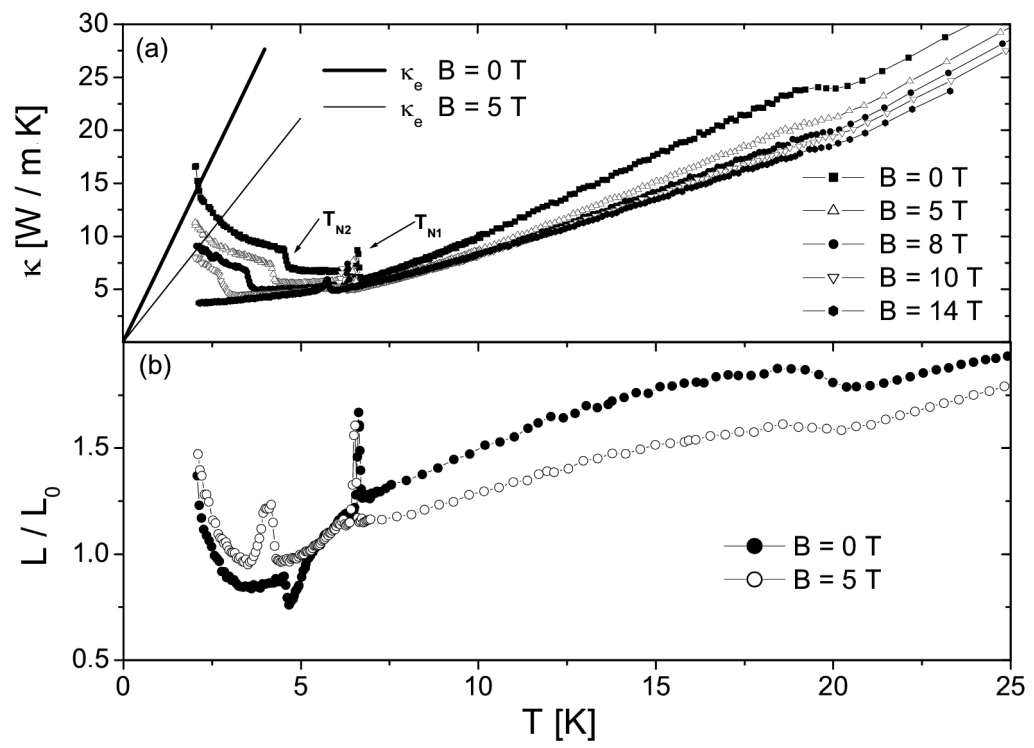

Fig. 1. (a) Thermal conductivity in the applied magnetic fields. Solid line presents the electronic contribution $\kappa_{\mathrm{e}}$, (b) Lorentz functions at $0 \mathrm{~T}$ and $5 \mathrm{~T}$.

of $B=5 \mathrm{~T}$. In order to confirm this expectation and to determine the dependence of $\kappa(T)$ below the maximum $\kappa \sim T$ (electrons) or $\kappa \sim T^{3}$ (magnons or phonons) [7], measurements at lower temperatures are necessary. The different observed low temperature behavior compared to this in [6] can be due to higher purity of our sample.

Figure $1 \mathrm{~b}$ shows the experimentally determined temperature dependences of reduced Lorentz number $L / L_{0}$ of $\operatorname{PrB}_{6}$ for magnetic field $B=0 \mathrm{~T}$ and $5 \mathrm{~T}$, where $L=\kappa \rho / T$ and $L_{0}=24.5 \mathrm{nW} \Omega \mathrm{K}^{-2}$. The values of $L / L_{0}$ are close to 1 at low temperatures, which is in agreement with the conclusion that the electronic part $\kappa_{\mathrm{e}}$ constitutes the main contribution to thermal conductivity. At about $20 \mathrm{~K}$ an anomaly occurs. According to [8], at temperatures of about $3 T_{\mathrm{N}}$ a critical divergence of the nuclear relaxation rate $1 / T_{1}$ ( $T_{1}$ represents the spin-lattice relaxation time) is observed, which may be inferred as an evidence for the development of short-range magnetic ordering. Whereupon we assume that the observed anomaly is related with this ordering. Moreover, the applied magnetic field gradually suppresses the anomaly, which reveals its magnetic origin. Further $L / L_{0}$ anomalies are observed at $T_{\mathrm{N} 1}$ and $T_{\mathrm{N} 2}$. Between them a sharp decrease in $L / L_{0}$ with decreasing temperature appears. This points out that the thermal conductivity is more largely than the electrical conductivity influenced (suppressed) by the inelastic scattering of electrons. Below $T_{\mathrm{N} 2}$ our values do not decrease as in [6], but an increase in $L / L_{0}$ occurs, which can originate from the contribution of the magnons to thermal conductivity in this temperature range. 


\section{Acknowledgments}

The authors acknowledge the support of this project by the Scientific Agency VEGA 7054, Science and Technology Assistance Agency APVT-51-031704, COST ECOM-P16 and INTAS 3036. U.S. Steel Košice, s.r.o., has sponsored a part of liquid nitrogen.

\section{References}

[1] J. Etorneau, J. Less-Common Metals 110, 267 (1985).

[2] J. Etorneau, P. Hagenmuller, Philos. Mag. B 52, 589 (1985).

[3] C.M. McCarthy, C.W. Tompson, R.J. Graves, H.W. White, Z. Fisk, H.R. Ott, Solid State Commun. 36, 861 (1980).

[4] K.N. Lee, R. Bachmann, T.H. Geballe, J.P. Maita, Phys. Rev. B 2, 4580 (1970).

[5] P. Burlet, J.M. Effantin, J. Rossat-Mignod, S. Kunii, T. Kasuya, J. Phys. (France) C 8, 459 (1988).

[6] M. Sera, S. Kobayashi, M. Hiroi, N. Kobayashi, S. Kunii, Phys. Rev. B 54, R5207 (1996).

[7] S.Y. Li, L. Taillefer, C.H. Wang, X.H. Chen, Phys. Rev. Lett. 95, 156603 (2005).

[8] S. Takagi, S. Itabashi, S. Kunii, T. Kasuya, J. Magn. Magn. Mater. 52, 267 (1985). 